\title{
Politique
}

\section{À l'ombre de la dynamo technologique : dépendance technologique, innovation et pôle de développement}

\section{Christian DeBresson}

Numéro 10, automne 1986

Opinions et votes

URI : https://id.erudit.org/iderudit/040534ar

DOI : https://doi.org/10.7202/040534ar

Aller au sommaire du numéro

Éditeur(s)

Société québécoise de science politique

ISSN

0711-608X (imprimé)

1918-6584 (numérique)

Découvrir la revue

Citer cet article

DeBresson, C. (1986). À l'ombre de la dynamo technologique : dépendance technologique, innovation et pôle de développement. Politique, (10), 55-90. https://doi.org/10.7202/040534ar d'utilisation que vous pouvez consulter en ligne. 


\title{
À L'OMBRE \\ DE LA DYNAMO TECHNOLOGIQUE: DÉPENDANCE TECHNOLOGIQUE, INNOVATION ET PÔLE DE DÉVELOPPEMENT
}

\author{
Christian DeBresson \\ Centre de Recherche en Développement Industriel et Technologique \\ et Université Condordia
}

En 1969, George Grant, penseur de philosophie politique canadien, publiait une série d'articles dans des magazines populaires tels que Saturday Night, This Magazine et Canadian Dimension qui furent rassemblés dans un recueil sous le titre: Technology and Empire: Perspectives on North America. Dans ces essais, écrits lors d'un séjour à Hamilton, il mettait en lumière certaines particularités des valeurs canadiennes. George Grant utilisait «l'éclairage violent» de la guerre du Vietnam pour révéler l'incapacité du Canada de s'émanciper intellectuellement et culturellement des visées homogénéisantes de la «dynamo technologique» des États-Unis.

* Nous voulons remercier le Professeur François Perroux, Miguel Wionczek, François Chesnais, Graciela Ducatenzeiler, Arthur Kroker, Bernadette Madeuf, Lynn Mytelka, Michel Duquette un lecteur anonyme et les participants aux séminaires de l'I.S.M.E.A. à Paris et de l'Atelier de recherche sur l'économie et la politique de la technologie au Canada de l'Université de Montréal pour leur généreux commentaires. Sans l'assistance de Brent Murray, nous n'aurions pas pu terminer ce travail. 
C'est à cette époque, où le public canadien apprit que l'industrie canadienne fabriquait le système au laser qui guidait les «bombes intelligentes» qui recherchaient la chaleur humaine comme cibles au Vietnam (Regehr, 1975). La complicité canadienne dans les viséès technologiques et les pratiques militaires d'une guerre dont la majorité de la population n'approuvait pas l'objet, se révéla un dilemne pour la nation.

«La suprématie de l'empire américain dans le monde occidental», expliquait-il, "fut importante pour le Canada non seulement à cause de leur proximité géographique et économique, lesquels posent des problèmes de simple survie à notre nation, mais dans un sens encore plus profond car la domination des États-Unis fut identifiée avec la victoire sans équivoque de l'esprit progressiste occidental " (Grant, 1969: 71 - notre traduction).

Prométhée vint au Canada sous les traits d'ingénieurs étrangers, d'abord britanniques, puis américains. Prométhée n'est pas apparu aux Canadiens comme la promesse endogène d'une libération mais tout de suite comme la menace de nouvelles dominations étrangères. "Progrès technique» et "étranger» furent dès le début liés ensemble par l'ambivalence d'un sentiment qui devait passer de la haine à l'admiration - ce que Behrman et Hymer appellent le syndrome d'amour et de haine (love-hate).

La critique de Grant ne portait que sur la dépendance culturelle. Aujourd'hui, en 1985, à l'époque des recherches sur la "guerre des étoiles», ses préoccupations paraissent toujours d'actualité. En 1969, elles trouvèrent un large écho au sein d'écoles de pensée et de disciplines de recherche fort diverses. Dans cet article, nous nous limiterons à poser la question de la dépendance technologique du Canada, en évaluant la capacité du pays à initier des changements dans sa culture matérielle sans approfondir par ailleurs les questions reliées à la dépendance culturelle.

Depuis la publication du texte de George Grant, le contexte international a bien changé. Le rôle impérial des États-Unis s'est modifié. Pour s'être empêtrés dans une expédition malheureuse au Vietnam, les États-Unis ne purent faire face à l'éclairage «violent » 
de contradictions sociales internes. Après l'effondrement du régime de Saigon, l'impérialisme américain dut se retirer avec tout son arsenal technologique. Militairement «vaincu», une fraction de la population américaine considère à toutes fins pratiques cette défaite comme plus salutaire qu'une éventuelle «victoire» (Commanger, 1985), alors que pour une autre fraction de l'opinion américaine, la débacle du Vietnam reste encore une honte qui cherche un exorcisme dans des fantaisies cinématographiques comme «Rambo» ou un exutoire dans un «revanchisme» qui limite pour l'heure ses prétentions hégémoniques à l'hémisphère américain. Entre temps, l'impérialisme russe, profitant de la «détente» et du repli de son principal rival, s'est lui-même empêtré militairement en Afghanistan, alors que son allié Vietnamien s'installait au Cambodge, révélant avec force, et jusque dans l'exemple polonais, les contradictions internes du totalitarisme d'état.

Dans le même temps, aux États-Unis, l'insécurité intellectuelle, voire morale saisissait une partie importante des industriels. Depuis la récession du milieu des années 70 , les entreprises multinationales américaines se sont partiellement repliées. Le déficit commercial américain s'amplifie. En 1985, les U.S.A. redeviennent un pays emprunteur pour la première fois depuis 1914. Mais surtout, les industriels commencent à douter de la suprématie technologique de leur propre pays: la «menace japonaise» apparaît comme le nouvel élément dynamique de la technologie mondiale. Les Japonais, connus comme des imitateurs, ont devancé les ÉtatsUnis, non seulement par leur compétence industrielle et leur maîtrise technique mais dans la délicate gestation culturelle, institutionnelle et économique qui constitue le dynamisme innovateur d'une société. D'après la majorité des indicateurs technologiques disponibles, ' le Japon conteste l'avance des États-Unis dès avant

1. Recherche-Développement, taille du marché des brevets, prise de brevets nationaux et à l'étranger, exportation/importations de produits intenses en R-D, nombre d'ingénieurs, etc., à l'exception des paiements de transfert. 
1971 à l'époque des mesures de Nixon sur la convertibilité du dollar, profite de la récession de 1974-79 pour les dépasser, et s'installe clairement en tête dans les années 1980 (OCDE, 1984). On murmure même que la cinquième génération d'ordinateurs serait japonaise. Le Japon envoie deux sondes à la rencontre de la comète de Halley pour étudier les débuts du système solaire; la Russie et l'Europe seront au rendez-vous; les États-Unis n'y seront pas, annulant leur projet de sonde au bénéfice de l'initiative de défense stratégique. Telle est la crainte qui mine aujourd'hui la confiance dans la «destinée américaine»: le symbole du succès occidental - la démocratie et l'esprit d'entreprise américains serait supplanté par un modèle asiatique.

Cependant au Canada, les implications d'une éventuelle redistribution de l'initiative technologique mondiale ne sont pas encore perçues. À cause de la proximité géographique, économique et surtout culturelle des États-Unis, les firmes multinationales apparaissent encore aux nationalistes canadiens comme la source principale de la dépendance technologique.

Historiquement, la politique du Canada semble alterner entre une phase d'invitation sans restrictions à toute technologie étrangère et une phase de nationalisme technologique étroit, qui préfere tout développement technique domestique aux techniques étrangères. Depuis la seconde moitié des années ' 60 jusqu'à maintenant, nous aurions vécu une période plus ou moins nationaliste. Pendant cette époque, s'est répandue chez nous l'idée de notre dépendance technologique des États-Unis dont les multinationales américaines seraient la cause évidente. Cette perception simpliste a aussi cours chez les trois principaux partis politiques fédéraux: libéral, conservateur et néo-démocrate - et tout particulièrement dans leurs sections ontariennes. En 1975, cette idée avait probablement cours dans le Conseil Privé du Premier Ministre Trudeau: un de ses membres, Michael J. Kirby, représentant officiellement le bureau du Premier Ministre du Canada dans un forum latino- 
américain, y défendit l'idée que son pays était «technologiquement dépendant ${ }^{2}{ }^{2}$

La source de ce diagnostic vient probablement du rapport Gray sur l'investissement étranger (1971) qui reposait sur une variante radicale de la thèse de la dépendance technologique: "le Canada devient captif de la technologie étrangère»(Gray, 1972, souligné par nous). Simultanément, le Conseil des Sciences du Canada parlait dans un rapport officiel d'un supposé manque d'innovation. À la fin des années '70, deux géographes industriels, John Britton et James Gilmour, en marge de leur propre discipline, publièrent au Conseil des Sciences un rapport controversé intitulé Le Maillon le plus faible: appliquant avec plus ou moins de bonheur l'appareil conceptuel propre à la littérature latino-américaine dépendantiste à la réalité canadienne, mesurée avec plus ou moins de rigueur, ils concluèrent au "sous-développement ", "au retard " et à la «déficience technologique» du Canada (Britton et Gilmour, 1978). En 1982, Glenn Williams, un politicologue, après avoir documenté systématiquement les clauses de restriction à l'exportation dans les filiales au Canada, alla plus loin: il franchit un pas de plus et conclua que le Canada était «dépendant technologiquement" (Williams, 1983).

Nous ne discuterons pas ici de la validité et de la cohérence de la notion de dépendance quand elle est appliquée à la technologie, ni de comment une telle dépendance se mesurerait. Nous n'avons

2. "Historiquement, le Canada a été un exportateur de matières premières et de biens semi-manufacturés. En conséquence, avant la Seconde Guerre Mondiale, le peu de technologie utilisée au Canada était concentrée dans les secteurs non industriels de l'économie tels que l'agriculture, les mines et les pêcheries. Qui plus est, la technologie industrielle importée était le plus souvent utilisée "telle quelle» par une industrie dominée par l'étranger, sans aucune modification pour s'adapter aux priorités nationales... Le résultat est que le Canada se développa peu en matière de science et de technologie nécessaire pour répondre aux besoins croissants de la nation. Ces dernières années, cependant, le Gouvernement Canadien a reconnu le besoin de développer les capacités scientifiques et technologiques domestiques afin... de réduire la dépendance technologique... » (Kirby, 1975, notre traduction). 
pas non plus la place ici d'argumenter pourquoi le concept de hiérarchie dans les pôles technologiques de développement nous paraît plus féconde que celle de «dépendance technologique».

En effet, Miguel Wionczek du Collège de Mexico, spécialiste latino-américain de la dépendance technologique, doute que le concept de «dépendance» soit très utile pour l'étude du développement technologique d'un pays comme le Canada. «Si l'on peut prouver, comme vous l'avez fait, qu'il y a un volume considérable d'invention et d'innovation domestique qui provient du transfert de la technologie étrangère alors il ne peut s'agir de dépendance technologique ${ }^{3}$ - certainement pas de "captivité de la technologie étrangère» comme le soutenait le rapport Gray en 1971. L'innovation industrielle canadienne atteste de la capacité des entreprises à adapter la technologie étrangère à des besoins locaux et de générer des solutions alternatives. ${ }^{4}$

Cependant un paradoxe subsisterait: comment une industrie innovatrice peut-elle générer une économie qui ne semble pas bénéficier de dynamisme technologique? Suffit-il d'établir qu'une économie soit innovatrice pour démontrer l'absence de contraintes technologiques? Nous le pensons pas. En particulier, il faut examiner

3. Communication privée du 17 février 1984.

4. Voir notre Innovation in Canada 1984. Dans 79 industries représentant $65 \%$ des échanges inter-industriels du Canada, un échantillonage stratifié aléatoire représentatif de répondants de différentes tailles et régions de répondants a été combiné avec la méthode de recensement des innovations significatives développée par Mansfield-Freeman-Townsend (lecture de 30 collections de journaux professionnels, d'associations industrielles de 1945 à 1979, enquête et réponse de 270 experts industriels, de toutes les sociétés performant de la R-D et détenant des brevets au Canada). $70 \%$ des firmes ensuite interrogées sur des innovations pré-identifiées ont répondu (en 1979, 732 répondants contribuant un total d'environ 2,000 innovations significatives (pouvant remonter à 1945). Pour chaque innovation, les variables couvertes concernent les premiers utilisateurs, les fournisseurs, les concurrents ainsi qu'un index de pondération quant au degré de nouveauté international. Il s'agit de la première enquête sur l'innovation qui soit représentative à la fois de l'industrie dans son ensemble et des innovations significatives, et, qui analyse les liaisons techniques en amont et en aval sur une longue période. 
le caractère de ces innovations et comment ces innovations s'agencent entre elles pour évaluer si elles sont source de dynamisme.

Depuis 1970, a eu lieu un dialogue de sourds au Canada, entre le Conseil des Sciences et son Conseil Économique et Social sur les avantages du protectionisme ou du libre échange, de l'interventionisme ou du laisser faire, de la concentration ou de la concurrence. Bien que ces débats soient restés largement axiomatiques, et visaient à appuyer des prises de positions idéologiques, on a commandité à cette occasion diverses enquêtes empiriques sur les multinationales (notamment étrangères), le commerce international du Canada, la recherche et développement, et enfin l'innovation. Les enquêtes sur l'innovation de ces deux organismes donnèrent des résultats convergents (DeMelto et al., 1980; Fortier, 1980; DeBresson et al., 1981, 1982b, 1984a) mais aucune de ces enquêtes n'a encore pu statuer sur les contraintes du développement technique au Canada, car elles ne considèraient pas, en particulier, la manière dont ces capacités innovatrices s'agençaient entre elles. C'est ce que nous ferons ici. Tant sur un marché ouvert que protégé, l'existence de pôles de développement technique permet des économies externes, induit des innovations et parfois, un dynamisme dans l'accumulation technique, alors que l'absence de tels pôles prive l'économie de dynamisme - malgré la présence d'innovations.

Dans cet article, nous insisterons donc sur ces aspects dynamiques provenant des interactions innovatrices répétées entre firmes, constituant des pôles autour desquels il y a accumulation synergétique de savoir faire technique.

Les limites de notre analyse sont les suivantes: il reste à en développer la dimension spatiale et régionale, à examiner le réseau des interactions innovatrices entre firmes - et non plus seulement entre industries - et à examiner la performance exportatrice des innovateurs. Il faudrait aussi tenir compte des accords de transfert 
technique entre les multinationales étrangères et canadiennes et leurs filiales.

Après avoir examiné les fausses perceptions les plus communes concernant l'innovation au Canada, nous examinerons dans une seconde partie la concentration de l'activité innovatrice dans le secteur énergétique et du traitement des ressources naturelles. Ensuite nous porterons un diagnostique sur les principaux pôles du développement technologique au Canada. Dans une quatrième partie nous avancerons des hypothèses sur les obstacles à une accumulation dynamique du savoir faire technique pour tirer, en conclusion, les implications politiques de notre analyse économique.

\section{Les fausses perceptions}

Les fausses perceptions suivantes sont largement répandues au Canada: manque d'esprit d'entreprise, investissement sans innovation, dépendance exclusive sur la technologie étrangère, le rôle universellement néfaste des multinationales d'origine étrangère. Chacune de ces perceptions doit être corrigées pour déplacer les interrogations sur un terrain plus pertinent.

\section{Le Canada manque-t-il d'entrepreneurs innovateurs?}

À partir d'un échantillon stratifié aléatoire dans les 79 industries principales du Canada, il nous a été possible d'estimer la proportion des entreprises innovatrices dans la population des entreprises industrielles. Cette proportion est relativement élevées à l'exception de quelques industries: postes de radio et de télévision, petits appareils électriques domestiques, textile et cuir. Ces exceptions confirment l'étude de Pierre Bourgault: Innovation et structure de l'industrie canadienne mais prouve que ses conclusions

5. Seul un recensement italien de l'Instituto Centrale di Statistica dirigé par Giorgio Sirilli du Consiglio Nazionale delle Ricerche nous permet une comparaison. 
ne sont guère généralisables qu'à ces quatre industries (Bourgault, 1972). Toutes les autres industries ont plus de $10 \%$ des entreprises qui introduisent des produits ou procédés nouveaux.

Pour une quinzaine d'industries, dont celles qui sont intensives en matière de R-D (directe ${ }^{6}$ ) mais aussi les industries de transformation de matières premières, nous estimons à 20-25\% la proportion des entreprises innovatrices. Ce ne sont pas les «esprits animaux" du capitalisme - pour reprendre une expression de Keynes - qui font défaut au Canada. On peut s'interroger, cependant, sur les problèmes de croissance des petites firmes innovatrices, sur la capacité de producteurs à façon, ou en petits lots, d'opérer une transition vers la production en ligne de grande série (Fortier, 1980; DeBresson et Lampel, 1982; Steed, 1983). Une structure industrielle où dominent de vieux oligopoles bloque l'entrée et la croissance de nouvelles firmes; les oligopsones semblent (dans les métaux non ferreux, par exemple) interdire les bénéfices des économies externes de se propager à l'extérieur des firmes dominantes.

Une hypothèse psycho-antropologique suggère que les Canadiens seraient des inventeurs mais non pas des innovateurs: ils laisseraient leurs inventions se développer «en exil» (Brown, 1967). Cette hypothèse n'est pas conforme à l'expérience (DeBresson, 1982a). Une autre analyse, s'inspirant de la thèse de Weber et Parsons, suggère que la structure familiale de l'entreprise canadiennefrançaise (et pourquoi pas juive?) obère l'entrepreneurship (Taylor, 1957). Cette hypothèse n'est ni conforme à la réalité Québécoise (Sales, 1979) ni à celle de la révolution industrielle anglaise où il a été démontré que ce sont des familles étendues (voire des clans), comme celui des Darby, qui furent les institutions sociales

6. "Directe», c'est-à-dire R-D de la firme elle-même; "indirectement ", une firme peut bénéficier de produits intenses en R-D (directe): par exemple une usine textile bénéficie de fibres synthétiques, de colorants et de machines la rendant "indirectement" intense en R-D. 
les plus adaptées à la transmission tacite des nouveaux paradigmes techniques (Wallace, 1982). Il semble qu'il en fut ainsi de Bombardier à Valcourt (DeBresson et Lampel, 1982).

L'analyse du type d'entrepreneurship au Canada est à poursuivre. Le présent travail de l'historien Michael Bliss promet d'y contribuer (Bliss, forthcoming). Mais il ne faut pas en attendre un verdict décisif. Alexandre Gerschenkron nous a clairement démontré que les développements économique et technologique étaient possibles sans tradition d'entreprise privée (Gerschenkron, 1962). L'entrepreneurship privé n'était absolument nécessaire ou central ni en Russie (d'avant et d'après 1917) ni au Japon. Ainsi au Canada, l'État a-t-il souvent joué le rôle de super-entrepreneur, mobilisant de vastes ressources et coordonnant de multiples efforts dans un but commun (Canadien Pacifique, Ontario Hydro, pipelines, uranium, Énergie Atomique du Canada, le projet de la Baie James, les sables bitumeux, enfin les télécommunications par satellites). S'il n'existait pas d'esprit d'entreprise au Canada - ce qui n'est pas le cas - les projets du gouvernement semblent pouvoir l'«induire». En fait, les «esprits animaux» répondent rapidement à ses sollicitations.

\section{Adoption de technologie étrangère et création technique}

L'accumulation du savoir faire technique constitue un continuum où l'adoption, l'imitation, puis l'adaptation, l'amélioration et l'innovation sont situées sur le même vecteur d'apprentissage technologique (Halty-Carrere, 1979). Un index du degré de nouveautés sur 2,000 innovations de notre enquête nous permet d'évaluer la position des firmes sur ce vecteur.

Comme Karl Marx, Thorstein Veblen, Nathan Rosenberg, Paul David, Richard Nelson et George Ray l'ont tous souligné: il y a des coûts, des risques d'échec et des maux de tête sérieux dans l'activité de pionnier. Aussi, contrairement à ce que suppose 
le concept d'entrepreneur-innovateur de Schumpeter, il est très rare que ce soit le pionnier qui tire lui-même des bénéfices d'un nouveau développement; le plus souvent, c'est celui qui ramasse les morceaux, après l'échec du pionnier, qui en profite. Car les seconds ont des coûts et des risques bien moindres dans l'adoption rapide des technologies mises au point par d'autres; mais ils bénéficient néanmoins des profits exceptionnels qui accompagnent le lancement des techniques.

En conséquence, on ne se surprendra pas de voir le Canada ne développer des "premières mondiales» que dans quelques domaines de spécialisation: la transmission haute tension d'électricité, le concassage et l'affinage des métaux non ferreux, l'exploration aérienne et magnétique des ressources minières, la fabrication de la pâte et du papier journal (où la machine à double tablier vient remplacer, après 150 ans, le système Foudrinier), la machine de scierie "Chip N' Saw» pour l'utilisation intégrale de la fibre de bois, les véhicules de transport hors route, la polymérisation Buta pour le caoutchouc synthétique, la fibre de verre pour les communications, la mécanique spatiale, les communications par satellite, l'insuline, l'énergie nucléaire à eau lourde, le laser $\mathrm{CO}_{2}$ TEA, etc... Cette liste partielle de "premières mondiales» démontre que le Canada sait générer des innovations radicales, et ceci, malgré le peu d'incitation économique qu'il y a pour une petite nation à promouvoir et encourager les activités pionnières des entreprises locales.

L'adoption rapide et rentable de technologie étrangère y est généralisée. Elle est habituellement associée à l'adoption de technologies d'âges variés. En général l'industrie canadienne est peu contrainte dans son choix de technologie étrangère - ni dans le pays d'origine ni dans son âge ( «vintage»). Au contraire, les firmes canadiennes sont souvent très agressives dans l'acquisition de technologies étrangères, y compris par le rachat de firmes étrangères (Niosi, 1982). Cette caractérisation générale des industries laisse 
évidemment place à des inégalités et recouvre des comportements micro-économiques variés: alors que certaines firmes font reculer les frontières techniques, d'autres, dans la même industrie, ont une stratégie d'application. Cependant, la majorité de nos 732 firmes examinées s'impliquent à la fois dans des "premières mondiales", des adoptions rapides d'innovations étrangères et des adoptions plus tardives, démontrant qu'au niveau micro-économique il ne semble pas non plus y avoir de contraintes rigides quant à l'adoption de techniques de différents âges. Les exceptions sont l'amiante (la filiale de Johns Mansville nationalisée par le Québec), les produits de caoutchouc et peut-être la chimie industrielle, où les Canadiens semblent contraints à adopter les innovations de leurs maisons mères.

Si nous évaluons la nature de l'activité innovatrice du Canada par une combinaison de critères (degré de nouveauté, nombre de pays d'origine, étendue - "scope» - des efforts techniques, ${ }^{7}$ capacité d'assemblage de systèmes entiers), tous les indicateurs convergent pour confirmer ce diagnostic. Les industries d'appareils de radio et de télévision, de l'automobile, d'appareils électroménagers, du textile et du cuir sont peu innovatrices. D'autres industries: - la chimie industrielle, le pétrole, les produits du caoutchouc, la fabrication des camions - sont spécialisées dans certains champs techniques seulement. Toutes les autres industries, par contre, s'approvisionnent auprès de diverses sources et développent des technologies dans une variété de domaines considérés comme essentiels à leur pratique industrielle, sachant aussi assembler des systèmes techniques entiers.

Il est vrai que l'essentiel de l'innovation au Canada se fait dans des industries où le rythme de l'accumulation est lent et progressif, sans sauts ou investissements massifs et indivisibles. ${ }^{8}$

7. Évaluée par les associations industrielles Innovation in Canada, pp. 67-69.

8. D'après l'analyse du ratio $\mathrm{r}=$ nouvelles machines par industrie de 1969 à 1979, Ibid. pp. 59-60. 
Ceci est particulièrement le cas pour le traitement des matières premières. Mais cette lente accumulation paraît elle soutenue et souvent induite par les investissements de l'État dans l'énergie, le transport et les communications; or ceux-ci sont massifs.

La vision du Canada en tant que pays qui se contente d'adopter de la technologie étrangère sans la modifier ni innover, ne semble donc pas conforme à l'expérience historique. Cependant, le fait que ce soit une perception très répandue dans le public est un facteur sur lequel il faut compter. La politique du Conseil Privé de Pierre-Elliot Trudeau reposait peut-être aussi largement sur cette fausse perception.

\section{Investissement étranger et oligopole international}

Le Canada - cela est connu - est le terrain préféré de l'investissement américain. Bien que l'impact de l'investissement direct des États-Unis au Canada ait été étudié sous presque tous les angles, nous avons examiné son impact particulier sur l'innovation industrielle. La proximité territoriale et culturelle du Canada permet à un dirigeant d'entreprise, américain à partir de son siège social de New York ou de Chicago, et avec quelques coups de téléphone, sinon un simple voyage de quelques heures, généralement le même jour, et - plus important - dans sa propre langue, de contrôler ses succursales canadiennes. Ces facteurs en font une partie intégrante de ses opérations nord-américaines. La part des investissements étrangers au Canada, bien que déclinante de 1974 à 1984, domine largement la chimie industrielle et l'automobile, par exemple. L'influence des investissements étrangers est très importante dans l'industrie manufacturière et représente du tiers à la moitié du total dans les industries primaires. Encore en 1971 , selon l'O.C.D.E., $64 \%$ des filiales y étaient astreintes à des clauses restrictives pour l'exportation (voir aussi Williams, 1983).

À cette époque, l'OCDE estimait que les multinationales contrôlaient $20 \%$ du commerce mondial et que leur production 
à l'étranger dépassait le montant total des échanges commerciaux internationaux. Ainsi, le rôle des oligopoles mondiaux était déterminant, au niveau général, dans l'organisation du marché mondial et, en particulier, pour un petit pays comme le Canada qui dépend dans une large mesure du commerce international.

La capacité de mobilisation financière de multinationales (étrangères ou canadiennes) permet d'investir simultanément dans les divers éléments techniques complémentaires, c'est-à-dire dans des systèmes techniques entiers. Ces systèmes de techniques interdépendantes vont ensuite induire la direction des recherches techniques. Cette capacité d'investissement est considérable en comparaison avec les capacités financières des gouvernements fédéral ou provinciaux du Canada. Leur capacité d'orienter le développement technique est donc centrale; leur capacité de contrecarrer les choix techniques nationaux ou provinciaux l'est aussi. Une multinationale comme MacMillan Bloedel, par exemple, planifie ses investissements à l'aide d'une matrice des échanges interindustriels internes, choisissant des structures de complémentarités et d'économies externes conformes à sa stratégie de marché. Ces préférences de structures ne seront pas forcément conformes à celles de la l'État-nation ou de l'État provincial. Une multinationale multiproduit peut être amenée, par exemple, à cause de surcapacités à l'étranger, à ignorer un coût plus bas proposé par un fournisseur domestique. On assiste à une préférence pour des liaisons au-delà des frontières, mais internes à son propre système productif. Il en résulte un jeu entre des préférences de structures, de complémentarités et d'économies externes divergentes, sinon antagoniques, des multinationales et des États, chacun aggrégeant des structures qui lui sont favorables et, par la force des choses, destructurant des structures rivales dans des espaces économiques de référence qui leur sont propres.

Depuis 1965, cependant, les préoccupations des nationalistes se sont plus portées sur les multinationales d'origine étrangère, 
en particulier celles d'origine américaine, que sur l'internationalisation des oligopoles et leur pouvoir. Les multinationales canadiennes comme Noranda, Inco, Abitibi, Alcan, Northern Telecom, Massey Ferguson, Bombardier, etc. participent à ce phénomène (Niosi, 1982). Comme l'ont justement signalé Litvak et Maule (1981), il y a un manque de rigueur des nationalistes lorsqu'ils ne portent leur attention que sur l'investissement étranger alors que les effets économiques des multinationales canadiennes sur l'économie canadienne sont quasiment identiques (Cordell, 1973).

Classifiant les entreprises d'après leur contrôle canadien ou étranger, nous avons pu comparer, dans chaque industrie, leur performance innovatrice. Les résultats ne permettent pas un diagnostic simple. Dans les industries où le Canada est très innovateur (pâtes à papier, métaux non-ferreux, sidérurgie, aéronautique, produits électriques, équipement agricole) les succursales étrangères sont aussi, sinon plus, innovatrices que les firmes sous contrôle canadien. Il n'y a que l'amiante, la chimie industrielle et les produits du caoutchouc où le contrôle de l'étranger semble avoir un effet négatif sur la performance innovatrice (selon l'âge des technologies introduites ou selon l'effet de préemption sur l'activité innovatrice domestique - Hirschman, 1958). Dans ces cas seulement, une politique de désenclavement, rompant le monopole étranger, paraît se justifier du point de vue du développement technique. Mais les effets bénéfiques ou néfastes des investissements étrangers (et des multinationales canadiennes) devraient être estimés plus précisément en fonction de leur intégration en amont et en aval. Sans anticiper sur les résultats d'un tel examen, il semble plus probable que le degré d'intégration et de retombées techniques est davantage lié au degré de rigidité des oligopoles et oligopsones qu'à l'origine nationale de ses participants. La plus grande intensité en R-D de certaines firmes canadiennes est compensée chez les filiales étrangères par des dépenses de transfert (Bones, 1979) sans impliquer forcément moins d'innovation technologique. 
Au terme de cette vérification expérimentale du bien fondé des perceptions populaires sur la «dépendance technologique» du Canada, et n'ayant trouvé aucun appui empirique à ces préjugés, nous sommes toujours confrontés à notre paradoxe de départ: comment une industrie innovatrice peut-elle être conciliable avec une économie qui ne semble pas avoir les attributs du dynamisme technologique.

\section{L'exubérance des ressources et une dynamique de croissance extravertie}

Si l'industrie canadienne est innovatrice, le manque de dynamisme technologique et économique ne proviendrait-il pas du type de spécialisation industrielle? Jorge Niosi pense que «le capitalisme canadien et sa bourgeoisie sont forts dans les secteurs technologiquement les plus simples et les plus traditionnels" (Niosi, 1982:207).

En effet, de même qu'il existe des industries qui sont plus industrialisantes que d'autres, de même il est des formes de technologies qui sont plus motrices de l'accumulation technique que d'autres. L'accumulation du stock de savoir faire technique se fait plus rapidement dans un pays qui possède des industries en biens d'équipement (Rosenberg, 1963a, 1963b, 1968, 1978; Saul, 1980; Freeman, 1963). Ceci est vrai tant pour les filières techniques bien établies que dans les lignées techniques nouvelles (informatique, bio-technologie, laser, nucléaire): ce sont les équipements qui vont incorporer les savoir faire techniques, en généraliser l'usage, capitaliser les savoir faire dans les ventes commerciales. Les firmes d'équipement institutionalisent les pressions en vue d'une diffusion technique. Le génie conseil peut mémoriser les expériences industrielles et l'apprentissage qui en résulte (Perrin et Real, 1976), mais seulement dans la mesure où ces leçons sont transformées dans de nouveaux équipements y aura-t-il capitalisation et avantage 
commercial du savoir faire accumulé. Le second secteur moteur de l'accumulation technique est celui des biens de consommation durable: entraînant des liaisons en demande finale, les liens de consommation permettent aussi le changement de la culture matérielle, c'est-à-dire une véritable transformation technique. Dans la hiérarchie techno-industrielle, ce sont là les deux formes industrielles de la technique qui sont le moteur de l'accumulation technique. Or ce sont aussi les deux aspects les plus faibles de la technologie au Canada.

Au Canada, le secteur des matières premières (récupération, extraction, transformation) fournit un quart des innovations et en utilise le tiers. Avec ses industries du bois, des métaux et de l'énergie, le Canada possède des industries parmi les plus innovatrices et sophistiquées au monde (intenses en R-D, capital et utilisation d'énergie). Si ces capacités industrielles et technologiques permettent au Canada de répondre stochastiquement à l'augmentation de la demande mondiale (Nijowhne S., 1979) ce ne sont pas, cependant, des industries motrices de l'accumulation technique.

Nous pourrions nous attendre à ce qu'une industrie innovatrice de l'équipement et de biens finaux existe au moins en amont et en aval de ces industries de traitement des matières premières, profitant d'une demande substantielle et des externalités d'une structure d'offre robuste. Mais l'innovation en biens d'équipement, même pour fournir ces secteurs de force, y est des plus modeste et inégale. De niveau mondial pour les équipements destinés aux industries du bois (derrière les Scandinaves), spécialisée dans ceux que l'on destine au secteur énergétique (turbines hydro-électriques, par exemple), l'innovation en biens de capitaux pour les métaux non-ferreux y est très faible - malgré le fait que des sociétés étrangères viennent s'installer au Canada pour venir y bénéficier de l'apprentissage technique auprès de clients qui sont des leaders mondiaux. 
Quant à l'innovation en biens de consommation, elle est rare au Canada: motoneige, articles de sport ou de luxe, fromage, alcool, bière. Les effets d'entraînement économique par les liaisons en demande finale et les occasions de modifier la culture matérielle des canadiens sont aussi rares.

La question qui se pose est la suivante: Pourquoi une telle concentration d'innovations dans les ressources? L'intuition du bon sens et la réponse de manuel économique suggèrent: les avantages comparés «naturels» («ricardiens»). Mais cette réponse n'est que partiellement satisfaisante: les «avantages absolus» de Smith - un peu oubliés par les manuels - nous permettent peut-être un peu mieux de comprendre cette concentration que les «avantages comparés» de Ricardo. Car l'abondance (ou la rareté) relative des facteurs ne peut «induire» l'innovation que par l'intermédiaire des attentes confiantes des entrepreneurs d'assister à des divergences régulières, et à long terme, dans la variation des coûts des facteurs (David, 1975); et ce sont les investissements qui s'en suivent, d'où proviennent des avantages comparés «acquis", qui sont les facteurs réels et directs qui induisent l'innovation dans cette direction (Schmookler 1966, 1972; Scherer, 1982; Séguin-Dulude et Amesse, 1984).

L'abondance des cours d'eau, la richesse du bouclier canadien et des Montagnes Rocheuses en cuivre, nickel, or, plomb, argent, cobalt, uranium, molybdenum, l'immensité des forêts ne furent que des opportunités qui, «interprêtées» par les investissements, ont «induit» l'activité technique dans cette direction. Ce sont les expectatives confiantes de pouvoir disposer, sur une longue durée, de ressources naturelles abondantes et accessibles qui ont orienté les décisions d'investissements dans cette direction. Nous précisons: ressources accessibles. Or l'accessibilité ne vint qu'après de grands travaux d'exploration, de transport et d'énergie. Les investissements massifs et "indivisibles» (lumpy) de l'État ont transformé des ressources inaccessibles en des ressources accessibles, 
induisant cette orientation technique. Les plus grandes découvertes minières se sont faites grâce à un quadrillage géologique gouvernemental, lors de construction des voies ferrées (par exemple cobalt) et grâce à la mise en place d'un réseau de transport aérien vers les communautés reculées dans les années 1920. De même l'histoire de l'exploitation forestière du Canada est celle de techniques de transport: chemins de fer, tracteurs désarticulés, souples et à quatre roues motrices (Radford, 1982); engins de coupe et d'élaguage des arbres; camions à remorques multiples sur voies uniques assistées de communications radiophoniques; grues mobiles; barges autodéversantes, etc. Dans le domaine hydroélectrique, la fabrication de turbines géantes, la transmission à haute tension de quantités immenses d'électricité (techniques où seuls les Russes rivalisent les Canadiens) et de multiples techniques de contrôle du réseau furent les conditions de l'exploitation des ressources hydrauliques lointaines.

Pour la première transformation des matières premières, l'influence des facteurs d'offre technique est encore plus évidente. La fibre de bois de l'Est canadien n'est pas similaire à celle de l'Ouest (ou du Sud-Est des États-Unis). La séparation de la lignine et sa décomposition nécessaire pour la production de pâte à papier requièrent des techniques adaptées qui ne sont pas toujours généralisables. De même, un minerai ne contient jamais la même composition de métaux dans la même proportion qu'un autre minerai; et même quand deux gisements de minerai ont la même composition métallique, les métaux y sont généralement réunis de manière différente. En conséquence, les procédés de séparation et de réduction doivent en général être adaptés, voire développés. Nous sous-estimons souvent le caractère unique, non-généralisable, voire idiosyncratique de certaines innovations technologiques (Atkinson et Stiglitz, 1969).

En d'autres termes, l' «exubérance» en ressources naturelles du Canada (selon l'expression de Nathan Rosenberg), par rapport 
à sa densité humaine, ne résulte pas en un foisonnement industriel et technique sans efforts et investissements considérables. L'idéologie néophysiocratique du Canada qui attribue sa richesse à l'abondance de son sous-sol, de ses lacs et de ses forêts, obscurcit la véritable source de cette richesse: savoir faire acquis et investissements en temps et capital. Sans ces facteurs d'offre, l'effet de la demande, tant souligné par la «staples theory» d'Innis, n'aurait pas pu jouer (Dow, 1985).

L'orientation de cette production de matières premières à haute valeur (par poids et volume) transportées sur de longues distances dans le marché mondial («staples» selon Innis), n'est donc pas uniquement dûe à l'«avantage comparé naturel» ni à une riche dotation en ressources naturelles. Les décisions de fournir des fourrures pour le marché de Londres et de Paris, de grands arbres pour la marine anglaise, du papier journal pour les ÉtatsUnis, du nickel pour les blindés de la première guerre mondiale, de l'uranium enrichi pour le projet Manhattan, des métaux non ferreux «précieux» pour le stockage stratégique des États-Unis durant la guerre froide - tous ces choix d'investissements furent le fait d'hommes d'affaires et de politiciens qui transformèrent un avantage comparé en un avantage acquis, parfois absolu et irréversible par l'accumulation de savoir faire et d'équipement. Inversement, leur décision de ne pas développer le savoir faire et l'industrie des biens de capitaux fut le résultat d'un choix conscient. En 1905, 25 ans après la "politique nationale» d'industrialisation, l'Association Manufacturière du Canada expliquait dans Industrial Canada une contre vérité: «naturellement la fabrication de machines est l'une des dernières industries à être développée dans un nouveau pays» (C.M.A., 1905: 717-18). Non seulement ce développement n'est pas automatique mais il précéda le gros du développement industriel en France, en Allemagne, aux États-Unis, au Japon aux Indes et en Chine (CNRS). Les responsables des pavillons canadiens dans les expositions internationales de Londres et de 
Paris écartaient sciemment les exemples de machines inventées au Canada pour exposer des objets techniques étrangers en usage au Canada, de façon à donner l'image d'un pays d'accueil idéal pour les immigrés technologiquement les plus sophistiqués. Encore aujourd'hui, il se peut qu'une division du travail implicite (ou explicite) restreigne les investisseurs canadiens de trop s'aventurer dans l'industrie de la machinerie - même quand celle-ci est destinée à des spécialisations canadiennes.

De même, les décisions de développer et diffuser des biens de consommation différents de ceux des États-Unis sont rares au Canada. La création d'une médecine sociale, amorçée en Saskatchewan en 1944, par un gouvernement social-démocrate puis généralisé dans l'ensemble du Canada par les Libéraux, est le seul cas de divergence importante dans la culture matérielle qui fut soutenue par les pouvoirs publics.

Cette orientation des élites dans le passé a créé son momentum d'inertie. Dans le temps (ordinal et) irréversible du développement technique, le passé contraint le présent. Ce qu'une firme peut entreprendre à un moment donné est conditionné par ce qu'elle a accompli techniquement dans le passé. L'activité passée suggère les problèmes techniques à résoudre, indique les opportunités nouvelles, établit un ordre du jour à la recherche. C'est alors que la contrainte du système technique, c'est-à-dire de l'interdépendance et de la complémentarité des techniques entre elles, et le poids des investissements passés convergent pour restreindre l'éventail du possible technique et de l'économiquement soutenable.

Il en résulte une «dynamique perverse» selon l'expression d'Aglietta. James Petersen, historien de la technologie des mines d'or ontariennes, explique que

«le processus de spécialisation qui commença aux États-Unis ... était ici (au Canada) souvent trop spécialisé tant régionalement qu'industriellement pour pouvoir fournir une base d'exportation de la technologie. Beaucoup de raffinements dans les techniques minières pour un sol gelé, développées dans le Yukon, furent oublicées lorsque l'activité minière s'effondra, alors que la Californie devint exportatrice 
de technologie minière après que les activités minières s'y étaient effondrées " (Petersen, 1977:60, notre traduction).

Notre propre enquête démontre que le plus souvent les équipements innovateurs y sont produits à façon ou en petits lots. Sans normalisation technique ni production en série, ces techniques ne peuvent le plus souvent être généralisées (DeBresson et Lampel, 1982a). Ehrensaft et Armstrong (1981) ont montré que cette "spécialisation extravertie» était commune dans les "capitalismes de dominion» (Australie, Nouvelle-Zélande, Afrique du Sud, Argentine, Uruguay). Dans cette dynamique perverse, la croissance des exportations s'est souvent accompagnée de réduction de la part des biens intermédiaires produits au Canada (Nijowhne et al., 1979:23). Le recul de l'intégration inter-industrielle peut aussi s'accompagner de disjonctions techniques et la perte des économies venant de complémentarités techniques. La diminution de biens de consommation distincts au Canada risque par ailleurs d'y entraîner l'assimilation aux États-Unis par le biais d'une culture matérielle nivelante.

\section{Les pôles de développement technique du Canada}

Devant une «dynamique perverse» et de possibles effets de disjonction, nous sommes amenés à rechercher les points d'ancrage du développement technologique du Canada. En 1978, le Conseil des Sciences du Canada nous commandait une étude qui visait à déterminer «s'il existait au Canada des complexes industriels, liant entre elles plusieurs industries innovatrices, formant des 'nœuds' de capacité technologique» ${ }^{9}$. Cet article tire le diagnostic résultant de cette étude empirique.

Nous articulons ailleurs les contraintes et les effets d'écodynamique qui poussent à l'émergence de "pôles» de développement

9. Termes du contrat, voir Innovation in Canada, vol. 1: page ii-iii. 
technique. Résumons-en ici les principales propositions. Nous savons que le développement économique est inégal dans l'espace et le temps. Il se fait par agglomération spatiale (pas forcément territoriale) et par à-coups. Pour nous, le développement technique est polarisé par une combinaison de contraintes:

(i) contraintes des systèmes techniques, c'est-à-dire de l'inter-dépendance de composants techniques;

(ii) les contraintes économiques de l'apprentissage, abaissant dramatiquement les coûts de transactions des interactions itératives entre fournisseurs et utilisateurs d'innovations;

(iii) l'exploitation des économies de variété (Baumol et al., 1982) avec des procédés de production en sur-capacité (Penrose, 1971);

(iv) les économies externes et les complémentarités de demande;

(v) les renforcements mutuels de systèmes techniques compatibles ou effets de jonction;

(vi) l'attraction du capital vers les foyers de profits exceptionnels.

Ces facteurs se combinent pour polariser le développement technique.

Quels sont ces pôles au Canada? Une analyse par graphes dirigés permet de les repérer sur une matrice triangulaire des interactions innovatrices: «cliques» ayant une densité maximum d'interaction, «complexes» techniques ayant des interactions avec une certaine transitivité et symétrie, et simple agglomérations d'interactions sans transitivité ni symétrie se distinguent à l'analyse.

Au Canada, il n'y a pas d'industrie qui joue le rôle de «nœud» technique (comme c'est le cas de l'équipement de transport en Angleterre), c'est-à-dire une industrie qui simultanément utilise des innovations de nombreuses industries et en fournisse à un grand nombre d'autres industries. L'industrie qui est la plus proche de jouer ce rôle est celle de la production et de la distribution d'énergie électrique. De grosses unités (Ontario-Hydro, HydroQuébec, B.C.-Hydro) y mobilisent des ressources technologiques considérables et variées. Les économies externes qui en résultent dans l'affinage des métaux non-ferreux, l'aluminium et les pâtes à papier sont évidentes (ces industries ont des taux d'utilisation d'énergie par tonne parmi les plus élevés du monde) mais les liaisons techniques en aval y sont parfois internalisées par les 
sociétés de transformation elles-mêmes (Alcan, Shawanigan Chemicals, Cominco, etc.). L'électro-raffinage et l'électro-métallurgie se sont développés au Québec. Récemment Hydro-Québec a essayé de promouvoir l'électro-chimie. En amont, le complexe électrique est appuyé par l'innovation du matériel électrique, des produits électriques et des moteurs divers. Le complexe est au cœur du système technique canadien.

Adossé au complexe énergétique, un complexe technique métallurgique et une agglomération technique de produits du bois constituent aussi des pôles dominants qui orientent l'activité technique au Canada. Le centre de gravité de ces activités techniques est l'énergie électrique. Ainsi Petersen explique que les ingénieurs furent incités à remplacer le travail humain dans les mines par la puissance hydroélectrique (op. cit.:61). L'historien économique de l'Ontario, H. V. Nelles relie le développement minier et l'exploitation forestière au mouvement pour une électrification décentralisée au début du siècle (Nelles, 1974). De même, l'industrialisation du Québec est marquée par les économies externes provenant de la sur-capacité des installations hydro-électriques.

Existe-t-il d'autres pôles importants? Les industries chimiques produisent beaucoup d'innovation mais sans intégration ou synergie technique apparente. Dans la région de Montréal, il existe une concentration d'activités innovatrices dans l'équipement de transport: aéronautique, matériel ferroviaire roulant et véhicules divers; mais sans intégration ou synergie innovatrice apparente et sans qu'il semble y avoir de fournisseurs de composants (freins par exemple) qui bénéficient de complémentarités de demande. La machinerie est aussi au Canada, comme dans tout pays un tant soit peu développé, un lieu privilégié de l'innovation mais il s'agit d'un secteur désarticulé. Quant au secteur de biens de consommation, l'activité innovatrice y est faible. Au bilan, il faut conclure qu'aucun des pôles de développement technique au Canada ne sont des pôles moteurs de l'accumulation technique. 
Pourquoi certaines agglomérations d'activités techniques et innovatrices n'ont-elles pas débouché sur des "effets de jonction" et de complémentarité, alors que d'autres ont entraîné une accumulation synergétique? Le cas le plus surprenant est celui de l'équipement de transport. L'investissement dans le transport a été une priorité nationale jusqu'en 1950. Dès 1840, le Canada produisait du matériel ferroviaire roulant. Et pourtant, le secteur du matériel de transport est encore aujourd'hui (1978) techniquement désarticulé. De nombreux facteurs économiques nous auraient fait prévoir ce secteur comme un des pôles principaux d'innovation au Canada. Il n'en est rien. Un autre exemple d'opportunité marquée est l'absence d' 'effet de jonction» entre la puissance électrique et l'infrastructure du transport ferroviaire dans l'Est du continent Nord-Américain où les densités semblent justifier l'électrification du réseau.

L'explication de ces opportunités manquées ne doit pas être recherchée, selon nous, dans une approche déterministe et uniquement dans les contraintes économiques et techniques. Les "pôles de développement technologiques» constatés en 1978 par notre analyse sont des points focaux de dynamiques d'accumulation et de modification de structures - non pas des structures stables. Bien que le poids des réalisations techniques passées implique un momentum d'inertie orientant l'activité de recherche technique aux alentours des champs d'action antérieurs, la jonction entre deux composants et systèmes techniques complémentaires peut créer par synergisme un nouveau pôle d'activité. Dans ce cadre, la présence (ou l'absence) de politiques de coordination d'investissements publics et de règlementations des gouvernements peuvent faire toute la différence entre l'existence d'un pôle dynamique d'accumulation technique et une simple agglomération désarticulée d'activités. La genèse de pôles est en partie une affaire de stratégie et de volonté publique. 


\section{Blocages internes et dépendances volontaires}

Dans les sections précédentes, nous avons conclu à une économie innovatrice sans accumulation dynamique. Il convient à présent de s'interroger sur les causes de cet état de fait. Les tentatives d'innovation qui ne furent pas poursuivies nous indiquent la nature des blocages, sans que nous puissions mesurer la contribution relative de chacun de ces facteurs de bloquage ou de la possibilité de les surmonter - ce qui ne peut à présent qu'être l'objet de spéculation.

Nous attribuons le manque de dynamisme technologique à l'exclusion des biens d'équipement et de consommation durables des filières de production les plus fortes au Canada. Nous avons déjà mentionné l'attitude des élites vis-à-vis des investissements dans ces secteurs. En général le peu d'attention porté par les gouvernements aux facteurs d'intégration et la manière dont les innovations s'agencent entre elles révèlent la faiblesse politique du projet collectif qui devrait se traduire par des choix délibérés et «un ensemble organisé et spécialisé d'économies externes» (Perroux, 1964). Il est vrai que la proximité spatiale (et territoriale) des États-Unis incite à profiter des externalités des investissements de ce pays (Rosenbluth). Il est toujours facile de téléphoner à Chicago pour faire réparer sa machine. Dans ce contexte c'est la transmissibilité même de la technologie étrangère (comme bien public) et la proximité d'un des foyers principaux de création technique (la «dynamo technologique» de Georges Grant) qui créent la possibilité de dépendances. Si un projet collectif national doit s'affirmer au Canada, les "préférences de structure" doivent être d'autant mieux articulées par les macro-décisions des pouvoirs publics pour compenser la force d'attraction des externalités des États-Unis sur les microdécisions des firmes. Or dans le domaine des biens publiques, par exemple, peu d'efforts ont été faits pour traduire des concensus sociaux quant au style de vie différent de 
la population par des normes et des standards spécifiquement canadiens. Sans rechercher pour autant à établir une divergence de systèmes techniques qui serait difficile de soutenir (l'expérience de CANDU l'atteste), une décision doit être prise pour savoir si le «Canadian way of life» en vaut la peine ou si la population canadienne, rassemblée dans sa majorité en marge du $49^{\mathrm{ème}}$ parallèle, doit se satisfaire d'être l'ombre du mode de vie de notre voisin au sud.

Par ailleurs, certains bloquages sont le fruit d'une attitude ambivalente vis-à-vis des oligopoles et de leurs pratiques restrictives. Le gouvernement du Canada se félicite de la présence de Northern Telecom et de l'Alcan qui fournissent plus de la moitié de la R$\mathrm{D}$ industrielle du pays. Et pourtant l'existence de ces deux sociétés multinationales canadiennes comme entités autonomes par rapport à A.T.T.-Bell et l'Alcoa est attribuable principalement à des décisions anti-trust du gouvernement américain. Le gouvernement canadien se garde bien, cependant, de tenter une telle politique anti-trust ou même d'utiliser, comme le font les Mexicains, la rhétorique de la loi anti-trust des E.U. pour interdire aux filiales américaines les clauses restrictives à l'exportation, à la substitution de composants, à la R-D et les augmentations de prix internes. Or la faiblesse de l'industrie de la machinerie pour les mines est certainement en partie attribuable à de telles pratiques restrictives de l'oligopsone minier. Beaucoup d'ateliers de machines sont captifs de groupes miniers, et quand ces derniers passent un contrat avec un fournisseur externe ils sont souvent tenus contractuellement de ne pas reproduire la machine en question pour aucun autre client. Bien qu'il soit justifié et assez fréquent que le premier client d'une nouvelle machine ait un droit prioritaire sur toute nouvelle version, voire des royautés (parce que le savoir faire qu'il a transmis au fabriquant doit aussi lui bénéficier) l'interdiction totale de reproduction d'une nouvelle technique est une pratique restrictive clairement néfaste à l'intérêt publique - qui 
ne tire avantage que de la diffusion d'une nouvelle technique et non pas d'une innovation exclusive non diffusée. Un autre cas de pratique restrictive obérant la diffusion d'une innovation concerne Caristrap, une petite multinationale canadienne qui produit un cerclage en polymer naturel très performant et peu coûteux se substituant au cerclages en acier, mais qui trouve sa croissance bloqué par les distributeurs de produits sidérurgiques.

Nous ne nions pas que les oligopoles innovent. Cette innovation se fait surtout en marge de l'industrie principale contre l'oligopole voisin: l'aluminium contre le cuivre et l'acier, le plastique contre l'aluminium, les produits de mais contre le sucre, l'automobile (General Motors) introduisant les diesels contre les fabriquants de matériel ferroviaire roulant, les fabriquants d'ordinateurs (IBM) introduisant une imprimante à laser sans pièces mécaniques mobiles contre les fabriquants de machines d'imprimerie, etc. Mais pour que cette concurrence de substitution, venant de l'extérieur à la Schumpeter, puisse se déployer, il est souvent utile de déserrer l'étau des vieux oligopoles pour faciliter les nouvelles technologies. Incohérences: les pouvoirs publics se félicitent des actions anti-restrictives des États Unis mais n'en prennent pas eux-mêmes. Il en résulte une dépendance sur les initiatives politiques des États-Unis. Comme l'a indiqué V. O. Marquez, ancien président de Northern Electric: "nous devons nous rappeler que ce sont les évènements de 1956 (action antitrust des USA) qui nous ont mis sur la voie d'un développement plus agressif, plus compétent, plus autonome et que cette voie n'était pas recherchée ou désirée par nous mais a dû nous être imposée» de l'étranger.

Un autre bloquage provient de la structure de l'accumulation au Canada qui semble privilégier les investissements financiers à l'investissement industriel. Nombre d'entreprises innovatrices, ayant des nouveaux produits commercialisés et des commandes en main, n'ont pu trouver les ressources financières de leur croissance 
et du passage à la production en série. L'absence d'aide financière au prototype et au lancement de la production est aussi un obstacle à de nombreuses innovations, alors que les bénéfices sociaux de la diffusion technique justifierait un mécanisme financier d'aide qui réduirait l'écart entre le prix acceptable pour le premier utilisateur et le coût du fournisseur pour les premiers modèles et permettent la démonstration de fiabilité de la technique. Trois banques canadiennes, pourtant, sont parmi les 10 premières mondiales (Moreau, 1981). Les capacités financières considérables du Canada s'investissent dans les prêts au jour le jour à Wall Street, la spéculation sur les commodités, les offres de rachat, les opérations foncières et la traite commerciale d'immenses mouvements de marchandise. Les banques canadiennes adoptent rapidement les toutes dernières innovations électroniques, qu'elles améliorent. Mais la force du système financier ne paraît pas soutenir le développement technique industriel; au contraire il le bloque souvent.

$\mathrm{Au}$ vu de ces cas de bloquages spécifiques, nous pouvons nous demander avec Kari Levitt, l'auteur de La Capitulation tranquille, s'il ne s'agit pour le Canada d'une dépendance ou d'une abdication en partie volontaire (Levitt, 1970). Wallace Clement a indiqué dans son The Canadian Corporate Elite que la bourgeoisie canadienne était divisée en trois groupes:

(i) un intermédiaire comprador,

(ii) un groupe contrôlant la sphère de la circulation,

(iii) un secteur domestique de services et de sous-contrat (Clement, 1977).

Les ententes avec la puissance industrielle des États-Unis se feraient, selon lui, directement par des négociations avec les sphères du capital au Canada qui contrôlent la circulation: banques, commerce, transport (Ibid. : 79). Ces secteurs du capital acceptent une division du travail et des zones d'influence dans l'espace économique nordaméricain et ne tiendraient pas tellement au développement d'un pôle au Canada centré autour de biens de capitaux fixes et de 
biens de consommation qui rivaliseraient avec des concurrents américains.

Au terme de cette spéculation sur les causes du manque de dynamisme technologique, il faut considérer l'influence des valeurs culturelles. Léopoldo Zéa a illustré une certaine forme de dépendance culturelle des élites latino-américaines vis-à-vis de l'Europe et les États-Unis. Ces élites n'ont aucune considération pour leur spécificité nationale, qu'elles dénigrent souvent, et désirent avant tout être des membres cosmopolites d'une métropole étrangère (Miami, New York, Paris). Ces élites choisiront la technologie étrangère par choix même quand la capacité technologique domestique est équivalente. L'internalisation des valeurs étrangères et le refus de développement d'alternatives propres par les élites ouvrent la voie à la dépendance.

\section{Conclusion: Implications Politiques}

1984: le Président des États-Unis, Ronald Reagan, communicateur de talent à la télévision adapte une image cinématographique de science fiction, dite «la guerre des étoiles», pour entraîner l'Occident dans une nouvelle vague de recherche et de développement à caractère militaire. Hiver 1985: presqu'immédiatement, les représentants du Pentagone font des conférences dans toutes les grandes villes canadiennes pour expliquer aux hommes d'affaires du Canada comment obtenir des contrats du nouveau programme. Après bien des hésitations gouvernementales, dûes aux doutes sur la fiabilité technique et stratégique d'une "guerre des étoiles», les sociétés privées et les chercheurs canadiens seront libres individuellement de participer ou non à l'initiative de la présidence américaine sans engagement propre du gouvernement fédéral canadien.

Nous re-voilà donc à notre point de départ, avec le même dilemme. Les questions de George Grant résonnent encore. L'en- 
treprise militaire de la métropole impériale nous entraînera-t-elle à nouveau dans des développements techniques que nous ne souhaitons pas? Ne risquons nous pas de mobiliser nos ressources techniques limitées dans un projet technique douteux alors qu'une collaboration technique avec le Japon serait aujourd'hui, par exemple, plus avantageuse? L'industrie d'armement du Canada, est déjà démesurée par rapport à notre industrie manufacturière, et une des premières exportatrices sur le plus grand marché militaire du monde dans le cadre d'un accord de production conjoint et de libre échange établi en 1939 et renouvelé en 1959, mais elle a très peu de retombées dans l'industrie civile (Reghr et al., 1981). Les innovations militaires sont de nature trop «baroques", complexes et sophistiquées pour être fiables dans la production civile (Kaldor, 1981). Pourtant la participation canadienne dans la R-D de la "guerre» dite "des étoiles" risque d'établir une hiérarchie de priorités susceptibles d'accaparer les capacités technologiques déjà limitées du pays.

Le gouvernement conservateur de Mulroney fut peut-être le crépuscule du nationalisme industriel et technologique. Les contrôles sur l'investissement étranger, par ailleurs inefficaces, sont démantelés (refonte de FIRA). Certaines sociétés de technologie électronique de pointe comme Mitel sont rachetées par des intérêts anglais, alors que les Finlandais rachètent des sociétés d'équipement forestier, Boeing rachète de DeHavilland. Des négociations de libre échange avec les États-Unis se poursuivent. Le contexte se prête à ce que la politique technologique passe à l'autre extrême: accepter toute technologie étrangère sans discrimination.

Notre analyse débouche évidemment sur un certain nombre de recommendations d'ordre politique quant à la politique technologique. Un examen des instruments politiques précis n'a cependant pas sa place ici. Notre philosophie ne nous incite ni à proposer des mesures améliorant le climat de l'entrepreneurship $\mathrm{ni}$, pour autant, des grands programmes interventionnistes de 
l'État. Par contre, prêter attention à la manière dont les innovations s'agencent entre elles, maximiser les effets de jonction et le degré d'intégration technique des pôles de développement nous semble pouvoir en être l'objectif raisonable d'une politique. Dans ce cadre, et dans la mesure où la volonté existe d'y investir les ressources et d'y adapter les institutions, il devrait être possible et économiquement soutenable de développer trois nouveaux pôles: le premier par l'intégration du secteur du matériel de transport; le second pour les équipements (instruments, machines, matériel de transport) s'adossant autour des points de force existants (énergie, métallurgie, bois); le troisième pour les biens de consommation qui fondent la distinction de notre culture matérielle. Pour ce dernier, le Conseil des Sciènces avait recommandé en 1978 que le pacte de l'automobile soit renégocié de telle sorte à attribuer au Canada une spécialisation dans les véhicules commerciaux, spéciaux et récréatifs, nous laissant l'opportunité de développer des véhicules hors route plus adaptés aux terrains variés et aux saisons extrêmes du grand nord que les véhicules de tourisme. Cette spécialisation est désirable tant dans un contexte de libre échange que protectioniste. Aussi, sélectivement, des normes et standards canadiens devraient exiger que les biens publics soient conformes à la qualité de la vie désirée.

Les pouvoirs publiques devraient aider à l'évaluation des innovations dont ils vont favoriser la diffusion. Les innovations ont en effet des caractères très variés, mais toutes ne sont pas facteur de croissance. À une époque où le "laisser innover» a remplacé l'idéologie du "laisser faire», il ne s'agit pas de limiter les initiatives innovatrices; bien au contraire: le pluralisme des centres innovateurs est socialement désirable. Mais, au-delà de l'innovation, les pouvoirs publiques ont la responsabilité de déterminer quelles innovations vont être favorisées dans leur diffusion par les normes, les standards, les règlementations diverses et les investissements publiques et quels vont être les systèmes techniques 
dominants. Dans le marché imparfait de l'information technique, les pouvoirs publics ont un rôle charnière dans la démocratisation des choix techniques pour rendre les acteurs économiques à même d'évaluer les diverses innovations disponibles.

Selon nous le rôle des pouvoirs publiques dans l'orientation de la technologie et des micro-décisions des investisseurs doivent surtout être de permettre l'émergence de consensus et de larges majorités autour de choix collectifs de la Cité. Comme les droits et prérogatives techniques n'ont pas été définis dans nos démocracies (selon Veblen), il conviendrait de promouvoir un espace politique dans lequel les citoyens puissent participer à l'évaluation sociale des choix techniques, de permettre la naissance de consensus et la diffusion des choix collectifs dans le respect des minorités. La politique de développement scientifique et technologique est née à la fin de la Seconde Guerre Mondiale comme sous-produit d'initiative militaire; il s'agit à l'heure actuelle de faire rentrer cette politique technologique dans le processus démocratique. L'encadrement du changement technique des pouvoirs publiques se fait, implicitement ou explicitement, à travers les règlementations, normes et standards ainsi que par les investissements et travaux publiques. Tous les pays ont commencé à se doter de mécanismes pour l'évaluation sociale des alternatives techniques (évaluation des performances techniques, analyses coûts/bénéfices, analyses d'impacts sociaux). En l'absence de tels mécanismes de démocratisation des choix techniques, la politique technologique canadienne continuera à alterner entre une attitude, à une extrême, qui trouve que tout développement domestique est meilleur qu'une technologie étrangère ou à l'autre extrême, l'invitation et l'accueil sans restriction à toute technologie étrangère quelle qu'elle soit et d'où qu'elle vienne. Dans un cas comme dans l'autre, la question pertinente, pourtant, n'est pas tant l'origine nationale d'une innovation, mais quel type d'innovation est choisi, sa compatibilité et sa complémentarité avec d'autres innovations et, partant, quel sera le système 
technique dominant qui en découlera. Il faudrait déplacer les débats de la politique technologique et, sur un autre terrain, cesser le dialogue de sourds auxquels nous ont habitués le Conseil Économique et Social et le Conseil des Sciences, pour placer les discussions sur le terrain de l'évaluation des alternatives. Ainsi, pourrons-nous dégager des consensus ou de larges majorités, fondant des choix collectifs.

\section{RÉFÉRENCES}

AGLIETTA, M. et R. BOYER (1985), «Pôles de compétivité, stratégie industrielle et politique macro-économique, \# 8222: CEPI-CEPREMAP, Paris.

ATKINSON, A. B. et J. E. STIGLITZ (1969), "New View of Technological Change», Economic Journal, Vol. XXIX, Sept., pp. 574-575.

BAUMOL, W. J. et al. (1982), Contestable Markets, New York, Harcourt Brace Jovanovich. BLISS, M., History of Entrepreneurship in Canada, (forthcoming).

BONES, H. P. (1979), "Assessing the Technological Capacity of Domestic and Foreign Controlled Firms in Canada", Paris", O.C.D.E.

BOURGAULT, P. (1972), Innovation and the Structure of Canadian Industry, Ottawa, Science Council of Canada.

BRITTON, N. H. et J. M. GILMOUR (1978), The Weakest Link: a Technological Perspective on Canadian Industrial Underdevelopment, Ottawa, Science Council of Canada.

BROWN, J. J. (1967), Ideas in Exile, Toronto, McClelland and Stewart.

CANADIAN MANUFACTURERS' ASSOCIATION (1905), Industrial Canada, Toronto, C.M.A.

CLEMENT, W. (1975), The Canadian Corporate Elite: an analysis of economic power, Toronto, McClelland and Stewart.

C.N.R.S./I.C.O.T.E.C. (1979), L'Adoption des techniques par les pays non-initiateurs, Paris, C.N.R.S.

COMMANGER' H. S. (1985), "Vietnam's Five Lessons for America, International Herald Tribune, May.

CORDELL, A. (1973), Essays on Aspects of Resource Policy, Ottawa, Science Council of Canada.

DAVID, P. A. (1975), Technical Choice innovation and economic growth: essays on American and British experience in the nineteenth century. London, Cambridge University Press.

DeBRESSON, C. (1981), "How Does Defence Contracting Affect Technological Capabilities of Canadian Firms?", in Regehr, E. (1981), Appendix D 1.1.

, (1982a), "Have Canadians Failed to Innovate? The Brown thesis revisited", Scientia Canadensis, Vol. VI, \# 1, pp. 10-23.

, et J. LAMPEL (1982b), «Bombardier's Mass Production of the Snowmobile the Canadian Exception?», Ottawa, Association canadienne d'histoire, dans Scientia Canadensis, automne 1985, no. 19: 133-149. 
,et B. MURRAY (1984a), Innovation in Canada, (2 vol.), New Westminster, CRUST.

DEMELTO, D., K. MULLEN et R. WILLS (1980), Innovation and Technological Change in Five Canadian Industries, Ottawa, Economic Council of Canada.

DOW, A., "Prometheus in Canada: Technological Change and the Expansion of Metal Mining, 1900-1950", in Fetschrift in Honor of Irene Spry, (à paraître).

EHRENSAFT, P. et W. ARMSTRONG (1981), "The Formation of Dominion Capitalism: Economic Truncation and Class Structure», in Moscovitch, Allan and Drower, G. Len, Inequality: Essays on the Political Economy of Social Welfare, Toronto, University of Toronto Press.

FORTIER, C. (1980), «Industrial Strength Through Innovation», Ottawa, Science Council of Canada, Annual Report.

FREEMAN, C. (1963), "The Plastic Industry: a Comparative Study of Research and Innovation", National Institute Economic Review, No. 26, pp. 22-62.

GERSCHENKRON, A. (1965), Economic Backwardness in Historical Perspective, Cambridge, New York, Praeger University series.

GRANT, G. (1969), Technology and Empire; Perspectives on North America, Toronto, House of Anansi.

GRAY, H. (1972), Foreign Direct Investment in Canada, Ottawa, H.M.S.O.

HALTY-CARRERE, M. (1979), Technological Development Strategies for Developing Countries, Montreal, I.R.P.P.

HIRSCHMAN, A. O. (1958), The Strategy of Economic Development, New Haven, Yale University Press.

KALDOR, M. (1981), Baroque Arsenal, New York, Hill and Wang.

KIRBY, M. J. L. (1975), "Science and Technology Programs in Canada», in Ingerson, E., Bragg, W. C., editors, Latin American Forum on Scientific and Technological Policy, University of Texas at Austin.

LEVITT, K. (1970), Silent Surrender; the Multinational Corporation in Canada, Toronto, MacMillan of Canada.

LITVAK I.A. et MAULE C.J. (1981), The Canadian Multinationals Toronto: Butterworths.

LOWER, A. R. M. (1938-1968), The North American Assault on the Canadian Forests, New-York, Greenwood Press.

MOREAU, F. (1981), Le Capital Financier Québécois, Éditions coopératives Albert SaintMartin.

NELLES, H. V. (1974), The Politics of Development: Forests, Mines \& Hydro-Electric Power in Ontario 1849-1941, Toronto, MacMillan of Canada.

NIJOWHNE, S. (1979), "Structural Change in the Canadian Economy», Ottawa, Statistics Canada, Structural Analysis Unit.

NIOSI, J. (1982), Les Multinationales canadiennes, Montréal, Boréal Express.

O.C.D.E. (1984), «Indicators of the Technological Position and Performance in O.C.D.E. Member Countries During the Seventies", Paris, DSTI/SPR 84.43.

PENROSE, E. (1971), The Growth of Firms; Middle East Oil and other essays, London, Cass.

PERRIN, J. et B. REAL (1976), L'Industrie des biens d'équipement mécaniques et l'engineering en France et Allemagne de l'Ouest, Grenoble, IREP.

PERroUX, F. (1964), L'Économie du XX ème siècle, Paris, Presses Universitaires de France. 
PETERSEN, J. O. (1977), "The Origins of Canadian Gold Mining", Ph.D. Thesis, University of Toronto.

RADFORTH, I. (1982), "Woodworkers and the Mechanisation of the Pulpwood Logging Industry in Northern Ontario, 1950-1970», Historial Papers, pp. 71-102.

REGEHR, E. (1975), «Making a Killing: Canada's arms industry», Toronto, McClelland and Stewart. , (et al.) (1981), The Utilization of Resources for Military Purposes in Canada and the Impact on Canadian Industrialization and Defence Procurement, New York, U.N. Disarmement and Development Study.

ROSENBERG, N. (1963a), "Technological Change in the Machine Tool Inquiry, 18401910», Journal of Economic History, Vol. XXIII, 14, pp. 414-443.

, (1963b), «Capital Goods, Technology and Economic Growth», Oxford Economic Papers, Vol. 15, pp. 217-227.

, (1968), «Economic Development and the Transfer of Technology: Some Historical Perspectives", Technology \& Culture, Vol. 11, pp. 550-575.

, (1978), "Learning by Using" in Inside the Black Box, Cambridge University Press.

ROSENBLUTH, G. (1957), Concentration in Canadian Manufacturing Industries, Princeton, Princeton University Press.

SALES, A. (1979), La Bourgeoisie industrielle au Québec, Montréal, Presses de l'Université de Montréal.

SAUL, S. B. (ed.) (1970), Technological Change: The United States and Britain in the Nineteenth Century, London, Methuen.

SCHERER, F. M. (1982), "Demand Pull and Technological Invention - Schmookler Revisited", Journal of Industrial Economics 30 (3), pp. 225-237.

SCHMOOKLER' J. (1966), Invention and Economic Growth, Cambridge Harvard University Press.

, (1972), Patents, Invention and Economic Change: Data and Selected Essays, Cambridge, Harvard University Press.

SEGUIN-DULUDE, L. et F. AMESSE (1984), «Les Transferts inter-industriels de technologie au Canada", Montréal, Atelier de recherche, Université de Montréal.

STEED, G. (1983), Threshold Firms, Ottawa, Science Council of Canada.

TAYLOR, N. (1957), "A Study of French-Canadian as Industrial Entrepreneurs», Ph.D. Thesis Yale.

WALlACE, A. F. C. (1982), The Social Context of Innovation: Bureaucrats, Families and Heroes in the Early Industrial Revolution, Princeton, Princeton University Press.

WILliAMS, G. (1983), Not For Export: Toward a Political Economy of Canada's Arrested Industrialization, Toronto, McClelland and Stewart. 